\title{
A Review of Nitric Oxide for the Treatment of Glaucomatous Disease
}

\author{
Joah Aliancy · W. Daniel Stamer · Barbara Wirostko
}

Received: March 22, 2017 / Published online: June 5, 2017

(C) The Author(s) 2017. This article is an open access publication

\begin{abstract}
Glaucoma is the leading cause of irreversible blindness worldwide, affecting 64.3 million people. An estimated 60.5 million people are affected by primary open angle glaucoma globally, and this will increase to 111.8 million by 2040. The definition of glaucoma has evolved greatly over time. Although multiple risk factors such as ischemia, inflammation, myopia, race, age and low ocular perfusion pressure may play a role, intraocular pressure (IOP) is still the main risk factor we can easily identify and modify. Currently, both medical and surgical interventions aim to reduce IOP. Effective IOP reduction controls and prevents the progression in many cases of glaucoma. Although this multifactorial disease's true pathophysiology is difficult to elucidate, physiologic mediators including nitric oxide (NO) are being evaluated as novel ways to impact progression by both lowering IOP and improving optic nerve head perfusion.
\end{abstract}

Enhanced content To view enhanced content for this article go to http://www.medengine.com/Redeem/ DC48F0605CF3B9A2.

J. Aliancy · B. Wirostko $(\bowtie)$

Moran Eye Center, University of Utah, Salt Lake City, UT, USA

e-mail: Barbara.wirostko@hsc.utah.edu

W. D. Stamer

Department of Ophthalmology, Duke University, Durham, NC, USA
Latanoprostene bunod $0.024 \%$ is an emerging therapeutic agent that has shown promise in clinical trials. As a nitric oxide-donating prostaglandin F2-alpha receptor agonist, it has proven to effectively, and with good tolerability, reduce IOP in glaucoma and ocular hypertensive patients. Latanoprostene bunod capitalizes on NO's ability to modulate the conventional aqueous humor outflow system, directly improving outflow through the trabecular meshwork, Schlemm's canal and distal scleral vessels. Importantly, targeting the conventional outflow tissues with NO-donating drugs represents an opportunity to restore outflow function, which will most likely have a beneficial consequence of additional IOP-lowering effects with dampening of diurnal and other IOP fluctuations, the benefit of a healthy trabecular meshwork.

Keywords: Clinical studies; Glaucoma; IOP; Nitric oxide; Nitric oxide synthetase; Trabecular meshwork

\section{INTRODUCTION}

Glaucoma is the leading cause of irreversible blindness worldwide [1]. An estimated 60.5 million people are affected by primary open angle glaucoma (POAG) globally [2]. This figure is projected to increase to 79.6.0 million in 
2020 and 111.8 million by 2040 [1-3]. The definition of glaucoma has evolved greatly over time. POAG is established as a chronic neurodegenerative disease leading to peripheral visual loss that is followed by loss of central vision. Normal tension glaucoma (NTG), a subset of OAG representing upwards of a third of all cases of glaucoma especially in Asia, is characterized by identifiable optic nerve and retinal ganglion cell degeneration leading to measurable visual field loss in the absence of elevated intraocular pressure (IOP). Ocular hypertension (OHT) is defined as having elevated IOP in the absence of optic nerve damage or visual field defects. Currently, both medical and surgical interventions aim to reduce IOP, a key risk factor [4]. Effective IOP reduction controls and prevents the progression in many cases of POAG, NTG and OHT. While IOP remains an important risk factor, other risk factors include ischemia, inflammation, myopia, race, age and low ocular perfusion pressure [5-8]. Although this multifactorial disease's true pathophysiology is difficult to elucidate, physiologic mediators, including nitric oxide (NO), are being evaluated as novel ways to impact progression by both lowering IOP and improving optic nerve head perfusion. This article is a comprehensive review based on previously conducted studies of $\mathrm{NO}$ and does not involve any new studies of human or animal subjects performed by any of the authors.

\section{PATHOPHYSIOLOGY OF GLAUCOMA}

Glaucoma is best described as an optic neuropathy characterized by slow degenerative structural changes seen in retinal ganglion cells (RGC) and the optic nerve head. Progressive neuronal cell death leads to the characteristic changes that are observed clinically and measured both functionally and structurally [4, 9-11]. Moreover, patients can undergo a significant amount of nerve fiber layer degeneration prior to clinical detection of POAG [4]. The rate of cellular death of RGCs has been shown to correlate with the level of IOP [12]. Intraocular pressure, the only modifiable risk factor for glaucoma, is determined by the balance in aqueous humor production by the ciliary epithelium and elimination through the conventional trabecular meshwork (TM) system and the non-conventional uveoscleral tract. IOP is set by resistance to pressure-dependent outflow of the aqueous humor in the conventional outflow pathway. Increased resistance to outflow through the TM and Schlemm's canal (SC) in the conventional outflow pathway leads to elevated IOP, which may distend the optic nerve head and damage RGC axons at the level of the lamina cribrosa $[10,13,14]$.

\section{CURRENT MEDICAL TREATMENT AND UNMET TREATMENT NEED IN GLAUCOMA}

The ultimate goal of antiglaucoma therapy is to maintain a patient's visual function and quality of life through their lifespan [15]. Prior to the early 1990s, the natural history of glaucoma was not well defined. The Early Manifest Glaucoma Trial (EMGT), initiated in 1992 in Sweden, was the first large, prospective, randomized and controlled clinical trial to evaluate the effects of treatment versus non-treatment on early stage glaucoma. This trial was paramount in demonstrating that every $1 \mathrm{mmHg}$ of IOP lowering matters because of the preferential benefit seen in the treatment group that received betablocker and/or laser trabeculoplasty compared with no early intervention. Risk of progression decreased $10 \%$ with each $1 \mathrm{mmHg}$ IOP reduction from baseline. This study, which included both OAG and NTG patients, helped determine the $t$ treatment required to reduce eye pressure and helped researchers chart the natural history of the disease. It also supported the concept that low blood pressure was a risk factor in normal tension glaucoma $[16,17]$.

The Collaborative Normal Tension Glaucoma (CNTG) study attempted to answer a slightly different question-the role of IOP reduction in a subset of glaucoma patients who exhibited IOPs in the normal range. The study questioned IOP-dependent disease pathogenesis and progression, while examining whether IOP had a negative synergistic affect along with 
other etiologic and pathogenic factors. About half of the patients were able to achieve a $30 \%$ reduction in IOP from baseline with medical intervention and laser trabeculoplasty. With this reduction, visual field loss progression was slowed in the treatment group versus the control [18]. The CNTG study's primary contribution was demonstrating that IOP reduction is critical in altering the disease course in patients with a normal IOP. Further, important non-IOP-dependent prognostic factors were elucidated from the study, including female gender, history of migraines, disc hemorrhages present at diagnosis, race and family history. These factors correlated with a significantly increased rate of disease progression [18].

The Ocular Hypertension Treatment Study (OHTS) continued to advance our understanding and approach to glaucoma management in "glaucoma suspects" with elevated IOP. This study demonstrated that topical ocular hypotensive agents achieving a 20\% IOP reduction from baseline delayed or prevented the onset of POAG. OHTS also helped to establish a tolerable safety profile for topical ocular hypotensive agents and identified risk factors that predicted which populations would more likely proceed to develop POAG with definitive optic nerve damage. For example, African Americans were found to develop POAG at a higher rate compared with all other racial groups in the study, despite adjusting for baseline IOP. In the delayed treatment arm, an increased cumulative incidence of POAG was seen at 13 years: $22 \%$ in the delayed treatment arm versus $16 \%$ in the treatment arm. Also, participants in the delayed treatment arm demonstrated more structural and functional damage, higher rates of bilateral disease and a shorter time interval to developing POAG. Generally, OHTS helped stratify low- versus high-risk OHT patients to determine effective management strategies [19].

The traditional methods of lowering IOP include reducing aqueous production, increasing uveoscleral outflow and increasing trabecular outflow secondary to contraction of ciliary muscle. Beta blockers, carbonic anhydrase inhibitors and alpha agonists all work to decrease aqueous production at the level of the non-pigmented ciliary epithelium. Prostaglandin agonists are a unique class that effectively modulates and improves outflow through the non-conventional uveoscleral tissue through extracellular matrix remodeling. Muscarinics, like pilocarpine, are very effective at contracting the ciliary muscle, which pulls on elastic tendons connected to the TM and Schlemm's canal (SC). As a result, outflow increases because of opening spaces in the TM and preventing SC collapse. However, muscarinics are generally only used in special circumstances because of the side effects and short half-life requiring frequent dosing. The need for a conventional outflow-modulating drug that can directly improve outflow through the TM, SC and distal scleral vessels is warranted. Nitric oxide agonists and rho kinase inhibitors are two novel drug classes that affect this system directly. Importantly, targeting the conventional outflow tissues with NO compounds represents an opportunity to restore trabecular outflow function, yielding additional IOP-lowering and dampening of diurnal and other IOP fluctuations.

\section{AQUEOUS DYNAMICS AND GLAUCOMA}

The conventional outflow pathway is comprised of the TM, SC and distal scleral vessels as a conduit for aqueous humor drainage $[20,21]$. The functions of the conventional outflow pathway are to provide the primary passageway for aqueous humor out of the eye and to generate resistance that sets intraocular pressure and dampens IOP fluctuations. The inner portion of the TM acts like a filter, intercepting cell debris and reactive oxygen species (ROS) to ensure that the resistance-generating juxtacanalicular connective tissue (JCT, consisting of JCT-TM cells and inner wall of SC cells) is not physically obstructed. TM cells are involved in phagocytosis, enzyme and growth factor production and the production of extracellular matrix components, such as proteoglycans, collagen and elastic fibers $[22,23]$. The structural integrity of the TM is dependent on complex interactions between the trabecular cells 
and surrounding extracellular matrix. Unlike the unconventional (uveoscleral) outflow pathway, conventional outflow is pressure dependent. Structurally, a healthy and non-glaucomatous TM demonstrates significant compliance to IOP changes, regulating and maintaining IOP at a constant level of $<2 \mathrm{mmHg}$ of fluctuation over a lifetime $[24,25]$. Importantly, the conventional outflow pathway serves as the predominant route of aqueous drainage, accounting for $70-90 \%$ of aqueous outflow. Individuals with elevated IOP have a conventional outflow system that is malfunctioning and no longer capable of maintaining an aqueous outflow homeostasis. Recent studies suggest that this is due to increased rigidity in the TM and its extracellular matrix [26]. Since NO is a local mediator for improving and decreasing contractility in the conventional outflow tract, deficiency of NO and or dysfunctional NO signaling may be a root cause of increased TM rigidity [27, 28].

\section{NITRIC OXIDE PHYSIOLOGY}

NO was first discovered in the 1770s by Joseph Priestly, an English chemist and theologian, but was disregarded for medicinal purposes based on the belief that it was an air pollutant [29]. This compound was then basically ignored for centuries. In the early 1900s, with the use of nitrates (e.g., nitroglycerin) for angina, pharmacologists began to characterize the physiologic responses of various tissues to these compounds. The cardiovascular literature started to report NO's benefit to improve angina pectoris and reverse ischemia [30]. While impacting cardiovascular tissue relaxation, nitroglycerin was also found to have a relaxing effect on respiratory and gastrointestinal smooth-muscle tissues. With this knowledge, the clinical applications of nitrates expanded beyond angina and myocardial ischemia to the treatment of other contracted smooth muscle tissue states such as reactive airway disease and gastrointestinal spasms [29]. In 1992, NO was recognized as the Science "Molecule of the Year" [31]. For their groundbreaking work "concerning nitric oxide as a signaling molecule in the cardiovascular system," Drs. Furchgott, Ignarro and Murad were awarded the Nobel Prize in Physiology or Medicine in 1998. Their work helped establish the foundation of our present understanding of the critical role of NO in the body. With more widespread clinical exposure, additional information was gathered on the tolerance and dosing of NO compounds as therapeutics. NO is now recognized as the elusive endothelium-derived relaxing factor (EDRF) and as a potent vasodilator that impacts numerous systems throughout the body [32-34].

There are three nitric oxidase synthases (NOS) that produce $\mathrm{NO}$ in the body, all of which are encoded by different genes: neuronal NOS1, endothelial NOS3 and inducible NOS2 $[35,36]$. Working directly, NO activates soluble guanylyl cyclase to produce cyclic guanosine monophosphate (cGMP). Indirectly, high levels of NO can lead to the production of reactive oxygen species (ROS) and become cytotoxic. NOS, an intracellular P450 enzyme, oxidizes L-arginine to L-hydroxyarginine and then to NO and citrulline [37]. In addition to vasodilatory function, eNOS plays an important role in mediating vascular endothelial growth factor (VEGF)-induced vascular permeability and angiogenesis [38]. Animal studies utilizing eNOS gene deletion mouse models demonstrate decreased VEGF-mediated NO production [39]. VEGF receptors are found ubiquitously on the membrane of vascular endothelial cells. When exposed to VEGF, these receptors trigger a downstream cascade that increases the concentration of NO. In addition, iNOS and nNOS serve as important factors in immunity and neurotransmission, respectively.

Because of its unique gaseous properties, NO generated intracellularly diffuses through cell membranes to rapidly work on target tissues. Target cells respond by activating guanylate cyclase and generating cGMP. This cascade ultimately leads to marked smooth muscle relaxation [37]. Recent studies have revealed that NO is also capable of altering calcium-dependent potassium channel conductance. Such modulation results in channel membrane activation and hyperpolarization with lower calcium ions resulting in vascular smooth muscle 
relaxation [37]. This alternative pathway provides another effective mechanism to achieve the vasodilation mediated by NO.

NO plays a key role in many organ systems. It is involved in the vasodilation of smooth muscle in the cardiovascular, urogenital, respiratory, gastrointestinal and even immune system. NO also has a role in angiogenesis, platelet aggregation and the musculoskeletal system with regulating bone formation. The mechanism of action is through either direct and/or indirect means as previously mentioned [40]. NO has a dual role in the inflammatory process, with both excessively low or high concentrations leading to pathology [41]. It is interesting to note that at high concentrations, NO can be proinflammatory and pathologic while also having desired antimicrobial effects. As such, it has been utilized as an antimicrobial agent. With reduced concentrations of NO, organ tissue systems are maintained in a contracted, constricted and/or high resistance state. Low levels of NO may be due to endothelial dysfunction, eNOS mutations or impaired NO signaling pathways, which can in turn lead to pathologic vasospasm. Vasospasm as well as smooth muscle constriction contributes to systemic pathologies such as myocardial infarction, stroke, Raynaud's disease, migraines, endothelial dysfunction, pulmonary hypertension, erectile dysfunction and perhaps even glaucoma [42].

\section{FUNCTION OF NO IN THE EYE AND ITS POTENTIAL BENEFITS TO GLAUCOMA THERAPY}

All three NOS enzyme isoforms are expressed in ocular tissues. Since measuring the NO concentration is challenging because of its very short half-life, NOS is identified in tissues indirectly by monitoring the conversion of L-arginine to L-citrulline and NADPH diaphorose staining [43]. Studies attempting to identify the predominant isoform in the conventional outflow tract have shown that iNOS is the predominant form in the TM, likely because of the presence of macrophages, while eNOS is the isoform expressed by SC cells and macrophages found in the TM $[28,43,44]$. TM cells, being smooth muscle-like cells, rapidly relax and decrease their cellular volume via $\mathrm{K}$ channels in response to $\mathrm{NO}[45,46]$. These changes account for the improvement in the TM morphology, leading to an increase in aqueous humor outflow [46, 47]. Moreover, glaucomatous eyes show decreased NADPH diaphorose staining in the conventional drainage pathway, compared with age-matched controls, suggesting an NOS role in the etiology of glaucoma [48]. These findings are consistent with recent genetic studies showing that polymorphisms in NOS3, the gene that encodes eNOS, are associated with higher risk of glaucoma [49].

The mechanisms underlying systemic blood pressure autoregulation and the relationship between vascular endothelium and smooth muscle appears to be analogous with the resistance regulation that is established within the TM and the SC in the eye [50]. Localized eNOS within the systemic vasculature endothelium is shear-sensitive [51, 52]. The endothelium within the SC exhibits this same shear stress sensitivity and suggests a regulatory role of $\mathrm{NO}$ in aqueous humor facility and IOP maintenance [53-55]. Moreover, endothelial cells within SC function as a barostat, mediating an endogenous NO feedback loop that is sensitive to mechanical effects of IOP changes. Collapse or narrowing of SC leads to NO production by eNOS within the endothelia [56]. NO produced by SC at elevated IOPs can diffuse in retrograde fashion to relax TM while also working downstream to affect smooth muscle in distal vessels and thus lower IOP. In addition, eNOS-produced NO influences intercellular junctions and plays a major role in vascular permeability. This function of NO might contribute to trabecular outflow resistance by affecting the inner wall of SC $[57,58]$.

The third NOS enzyme isoform is the neuronal NOS, nNOS. Neuronal NOS, along with eNOS, plays an important role in ocular blood flow and can be found in the anterior segment innervating intrascleral vessels distal to SC [59]. It is also present ubiquitously within the posterior segment, and concentrations are found within the amacrine, ganglion, pigment 
epithelium, and outer and inner nerve fiber layers [60-63]. Retinal vasculature has been found to demonstrate a vasodilatory response to NO liberated from nitrergic neurons in in vivo animal studies. Also, NO derived from endothelium and perivascular nitrergic neurons appears to be an important regulator of ocular blood flow homeostasis, especially within uveal tissue $[56,64]$. Basal ocular blood flow regulated by NO formed by eNOS and nNOS has been confirmed by studies involving both human and nonhuman models. In one such study, choroidal, iris, ciliary, optic nerve head and ophthalmic arteries, as well as retinal vessels, were all found to be NO responsive [65]. In another study, optic nerve head blood flow in healthy subjects was found to increase with administration of an NO-donating agent, thus giving rise to a possible role of $\mathrm{NO}$ in improving ocular perfusion in NTG [66].

Taken together, eNOS, NO availability and normal endothelial function play an important role in normal ocular function, in both the anterior as well as the posterior segments. A careful balance is maintained between the endothelin-1 and NO concentration as both molecules act on smooth muscle cell targets [67]. Endothelial dysfunction with altered eNOS function can alter this homeostatic balance, leading to decreased production of $\mathrm{NO}$ and an increase in both reactive oxygen species and endothelin-1 biosynthesis. This imbalance results in augmented endothelin-1 activity, primarily vasoconstriction, and may lead to the pathologic features seen in many optic neuropathies, namely POAG and NTG [68-70]. Endothelial dysfunction is recognized as being associated with normal tension glaucoma, perhaps through altered optic nerve perfusion [71]. However, there appears to be a strong association also with OAG [72]. Exogenous NO was found to decrease IOP by increasing outflow facility [73]. Interestingly, $\mathrm{NO}$ concentration in the vitreous humor correlates with the type and severity of glaucoma [74]. Levels of cGMP, an indirect indicator of $\mathrm{NO}$, were found to be decreased in the plasma and aqueous humor of NTG patients [75]. In addition, impaired peripheral endothelium-mediated vasodilation appears to be closely associated with NTG [76, 77]. These findings suggest $\mathrm{NO}$ as a target for manipulation in the glaucomatous eye and in NTG specifically, where there may have added value in NO-based vasodilation, improvement of ocular perfusion and enhanced oxygenation of tissues. Beyond lowering IOP, NO could play a role in manipulating and improving vascular endothelial regulation in the eye.

\section{NITRIC OXIDE AS A THERAPEUTIC}

NO-donating moieties have become attractive additions to existing drug agents to enhance therapy. Enhanced vasodilation, antiinflammatory properties and antiplatelet activity are just a few of the various features an NO-donor moiety can offer. For example, it has been established that NO inhibits the aggregation of platelets [78, 79]. Thus, an NO-donating compound could assist in inhibiting coagulation especially in type 2 diabetics where eNOS and hence NO production from endothelial cells is defective [78, 79]. Accordingly, a novel antiinflammatory compound, NCX 4016 (2-(acetyloxy)benzoic acid-3-[(nitrooxy)methyl] phenyl ester), has been in clinical trials as a prototype of a series of NO-donating hybrid drugs that can be potentially used to combat atherosclerosis and ischemia. In humans, orally administered NCX 4016 was capable of releasing NO and displayed a wide range of beneficial antiplatelet activities in both type 2 diabetics and non-diabetic patients with atherosclerotic cardiovascular disease [80]. In addition, these NO-donating antiinflammatory compounds have also shown preclinical and clinical safety and efficacy as antineoplastic agents. NCX 434, an NO-donating triamcinolone acetate compound, has been evaluated for improved optic nerve head oxygenation and retinal vasculature vasodilation in the cynomolgus monkey model of glaucoma [80]. Lastly, given the mounting evidence for the role of $\mathrm{NO}$ in modulating aqueous outflow, and the unmet need for a TM/SC modulator, NO has become an attractive molecule to develop clinically for lowering IOP. 


\section{EFFICACY OF NITRIC OXIDE IN THE TREATMENT OF OCULAR HYPERTENSION}

\section{Clinical Trial Evidence}

Latanoprost has proven to be an effective first-line IOP-reducing agent for the management of open-angle glaucoma by lowering IOP, primarily through the uveoscleral system. Although effective, many patients over time will require additional therapies to achieve their IOP targets and to slow down the progression of glaucoma. Latanoprostene bunod (Bausch and Lomb) combines the leading antiglaucoma pharmacologic agent, latanoprost, with a novel NO-donating moiety. Several trials have been conducted to support the clinical use of NO as an effective treatment modality for glaucoma. The recently conducted US Food and Drug Administration (FDA)-required clinical development trials (phases I, II and III) are discussed below.

KRONUS, a phase I open-label study, evaluated the effectiveness of latanoprostene bunod $0.024 \%$ as an IOP-lowering agent in healthy Japanese subjects. The mean $24 \mathrm{~h}$ baseline IOP of the study eye was $13.6 \mathrm{mmHg}$ with a standard deviation of 1.3. In addition, a prominent 8 a.m. diurnal rise in IOP was noted in the study's subjects. In the study protocol, subjects were instructed to self-administer latanoprostene bunod $0.024 \%$ once daily at 8 p.m. for 14 days. The absolute change from baseline in sitting IOP was then assessed on day 14 at 8 p.m., 10 p.m., 12 a.m., 2 a.m., 4 a.m., 8 a.m., 10 a.m., 12 p.m. and 4 p.m. The mean 24 -h IOP reduction was $27 \%$ compared to the baseline measurements. Latanoprostene bunod $0.024 \%$ demonstrated a significant IOP reduction despite the subjects' low baseline IOP with an acceptable and expected safety profile. Moreover, latanoprostene bunod $0.024 \%$ was found to lower the IOP at all time points, particularly during the morning hours when a diurnal IOP rise is common. All adverse events were considered mild, with punctate keratitis and conjunctival hyperemia noted as the most common adverse events [81].
The VOYAGER Phase II study was the preliminary trial to establish the optimum concentration of latanoprostene for future clinical trials. This investigator-masked study randomized 413 POAG or OHT patients to five groups with varying concentrations of the study drug ranging from $0.006 \%$ to $0.040 \%$, including a latanoprost $0.005 \%$ group. A total of 396 subjects completed the trial in 28 days. The primary efficacy endpoint was the absolute change from baseline mean diurnal IOP. The study concluded that latanoprostene's efficacy was dose-dependent, and $0.024 \%$ was the lowest dose to achieve the largest reductions in diurnal IOP versus latanoprost $0.005 \%$. For this reason, proceeding clinical trials involving latanoprostene bunod utilize the $0.024 \%$ concentration. In addition, latanoprostene bunod was found to have a good safety profile, with minimal safety issues-neither pupil dilation nor blurred vision were reported as adverse events. The results of this study suggest that NO has minimal to no effects at the ciliary muscle and that the NO component of latanoprostene bunod primarily affects the TM and SC to achieve additional IOP reduction compared to latanoprost 0.005\% [82].

The CONSTELLATION Phase II study compared latanoprostene's efficacy over a 24-h diurnal time period versus timolol $0.5 \%$. Latanoprostene bunod was found to have a greater effect on IOP reduction during the nocturnal period. The baseline IOP (SD) was found to be 21.6 (2.8) and 25.7 (3.8) $\mathrm{mmHg}$ for daytime and nighttime, respectively. Post treatment, the mean 24-h IOP was reduced to 17.6 (2.5) and 23.2 (3.4) $\mathrm{mmHg}$ for daytime and nighttime, respectively. Latanoprostene bunod demonstrated a sustained IOP reduction during the $24 \mathrm{~h}$ period, while timolol was primarily effective during the daytime. However, no statistical significance was achieved between the two in regards to overall diurnal IOP reduction [83].

The APOLLO and LUNAR studies were designed as phase III multicenter, randomized, active controlled, double-masked trials and had similar and comparable findings to previous trials. APOLLO enrolled 417 patients with OHT and/or POAG into their intent-to-treat population. Both studies evaluated latanoprostene bunod $0.024 \%$ versus timolol $0.5 \%$ over 
3 months [84]. IOP recorded at all post-baseline visits was found to be significantly lower in the latanoprostene $0.024 \%$ group versus the timolol $0.5 \%$ group: $17.8-18.7$ versus $19.1-19.8 \mathrm{mmHg}$, respectively, with an entry mean baseline IOP of 26.7 (2.5) $\mathrm{mmHg}$. Secondarily, latanoprostene $0.024 \%$ demonstrated superiority over timolol in percentage of IOP reduction and number of patients below $18 \mathrm{mmHg}$. An extended open-label safety phase demonstrated that latanoprostene was not associated with safety or tolerability issues that would limit clinical utility. The LUNAR study had similar conclusions [85].

The JUPITER study confirmed an acceptable safety profile over a 52-week span in Japanese subjects with POAG or OHT. A total of 121 patients completed this single-arm study in which latanoprostene bunod $0.024 \%$ was given every evening with concurrent evaluation visits to monitor adverse events. A mean baseline IOP of 19.6 (2.9) $\mathrm{mmHg}$ was measured at week 4 of the study. Latanoprostene bunod $0.024 \%$ reduced IOP by $22 \%$ within the first 4 weeks and achieved $26 \%$ reduction by 52 weeks from baseline to 14.4 (2.7) $\mathrm{mmHg}$, suggesting that long-term treatment could provide sustained IOP reduction [86].

In summary, latanoprostene bunod $0.024 \%$ demonstrates promising efficacy and safety as an ocular hypotensive agent and offers a novel mechanism acting on the TM. Latanoprostene bunod $0.024 \%$ was as effective as both latanoprost and timolol monotherapy, offering an additional 1-2 mmHg of IOP reduction. Complimentary action between the uveoscleral and conventional aqueous pathways may explain the efficacy seen in the major clinical trials. With a safety profile similar to latanoprost, latanoprostene bunod appears to be a favorable therapeutic option for glaucoma management.

\section{THE FUTURE OF NO IN OCULAR THERAPY}

The role of nitric oxide in the human body, and now specifically in the eye, has been gaining attraction in basic and clinical research. Nearly all human systems rely on NO and its indirect or direct effects for homeostasis. The roles that NO and the NOS enzyme isoforms play in ocular disease pathology, especially glaucoma and retinal ischemia, continue to emerge. A better understanding of the synthesis of NO, its signaling pathways and its end organ effects is leading to the identification of relevant clinical applications in ophthalmology. The use of NO donors is already a relevant therapeutic approach to treat myocardial infarctions, bacterial infections, wounds and now elevated intraocular pressure. Compounding an NO-donating group to an already effective prostaglandin analog will enable us to gain a novel tool in our glaucoma management toolbox that works on the conventional outflow system, offers a new mechanism of action and is complementary to already existing IOP-lowering agents. A challenge that remain is categorizing NO's duration of efficacy and penetration as a topical agent. Also, NO has a very short half-life and may require increased frequency of use or a different formulation. The projected value as established at the bench does not appear to translate equally into the clinic. This may be due to issues with corneal penetration and the drug not being liberated in the tissue with adequate concentrations being delivered to the site of actions, both at the level of the conventional outflow tract and optic nerve head. Furthermore, limited efficacy may also be due to the more complex heterogeneity of glaucoma in general. We may discover that there are further subtypes of glaucoma more specific to the underlying pathology in the TM that may warrant further classification of who would actually benefit the most from an NO-donating agent.

In conclusion, $\mathrm{NO}$ and its isoform synthetase enzymes are becoming therapeutic targets of increasing interest. As researchers and clinicians working in the ophthalmic space, we learn and borrow from the myriad of cardiovascular and systemic literature and data to help combat elevated intraocular pressure and prevent the multifactorial progressive optic neuropathy we know as glaucoma.

\section{ACKNOWLEDGEMENTS}

The design and conduct of the study was supported in part by an Unrestricted Grant from 
Research to Prevent Blindness, Inc., New York, NY, to the Department of Ophthalmology \& Visual Sciences, University of Utah. During the peer review process, the manufacturer of the agent under review was offered an opportunity to comment on the article. Changes resulting from comments received were made by the authors based on their scientific and editorial merit. All named authors meet the International Committee of Medical Journal Editors (ICMJE) criteria for authorship for this manuscript, take responsibility for the integrity of the work as a whole and have given final approval for the version to be published.

Disclosures. B. Wirostko holds stock and is the Chief Medical Officer of EyeGate Pharmaceuticals, Inc. J. Aliancy and W. D. Stamer have nothing to disclose.

Compliance with Ethics Guidelines. This article is based on previously conducted studies and does not involve any new studies of human or animal subjects performed by any of the authors.

Open Access. This article is distributed under the terms of the Creative Commons Attribution-NonCommercial 4.0 International License (http://creativecommons.org/licenses/ by-nc/4.0/), which permits any noncommercial use, distribution, and reproduction in any medium, provided you give appropriate credit to the original author(s) and the source, provide a link to the Creative Commons license, and indicate if changes were made.

\section{REFERENCES}

1. Tham Y-C, et al. Global prevalence of glaucoma and projections of glaucoma burden through 2040 a systematic review and meta-analysis. Ophthalmology. 2014;121(11):2081-90.

2. Quigley HA, Broman AT. The number of people with glaucoma worldwide in 2010 and 2020. Br J Ophthalmol. 2006;90(3):262-7.

3. Congdon $\mathrm{N}$, et al. Causes and prevalence of visual impairment among adults in the United States.
Arch Ophthalmol (Chicago, Ill, 1960). 2004;122(4):477-85.

4. Quigley $\mathrm{H}$, et al. Optic nerve damage in human glaucoma: II. The site of injury and susceptibility to damage. Arch Ophthalmol. 1981;99(4):635-49.

5. Sommer, et al. Relationship between intraocular pressure and primary open angle glaucoma among white and black Americans. The Baltimore Eye Survey. Arch Ophthalmol (Chicago, Ill, 1960). 1991;109(8):1090-5.

6. Sommer A. Risk factors for open-angle glaucoma: the Barbados Eye Study. Arch Ophthalmol. 1996;114(2):235.

7. Tielsch JM. Hypertension, perfusion pressure, and primary open-angle glaucoma. Arch Ophthalmol. 1995;113(2):216.

8. Bonomi, et al. Vascular risk factors for primary open angle glaucoma: the Egna-Neumarkt Study. Ophthalmology. 2000;2000:1287-93.

9. Leung C, et al. Evaluation of retinal nerve fiber layer progression in glaucoma: a prospective analysis with neuroretinal rim and visual field progression. Ophthalmology. 2011;118(8):1551-7.

10. Caprioli J, et al. Comparison of methods to evaluate the optic nerve head and nerve fiber layer for glaucomatous change. Am J Ophthalmol. 1996;121(6):659-67.

11. Miglior S, et al. Correlation between the progression of optic disc and visual field changes in glaucoma. Curr Eye Res. 1996;15(2):145-9.

12. Ekstrom C. Elevated intraocular pressure and pseudoexfoliation of the lens capsule as risk factors for chronic open-angle glaucoma. A population-based five-year follow-up study. Acta Ophthalmol. 1993;71:189-95.

13. Quigley H, Green R. The histology of human glaucoma cupping and optic nerve damage: clinicopathologic correlation in 21 eyes. Ophthalmology. 1979;86(10):1803-27.

14. Heijl A, et al. Natural history of open-angle glaucoma. Ophthalmology. 2009;116(12):2271-6.

15. European Glaucoma Society treatment principles and options 2008. In: Terminology and guidelines for glaucoma. 3rd ed. Savona: DOGMA Srl; 2008. p. 117.

16. Leske MC, et al. Early manifest glaucoma trial design and baseline data. Ophthalmology. 1999;106(11):2144-53. 
17. Hyman L, et al. Natural history of intraocular pressure in the early manifest glaucoma trial: a 6-year follow-up. Arch Ophthalmol. 2010;128(5):601-7.

18. Anderson D, Study N. Collaborative normal tension glaucoma study. Curr Opin Ophthalmol. 2003;14(2):86.

19. Kass MA. The ocular hypertension treatment study. Arch Ophthalmol. 2002;120(6):701.

20. Goel M, et al. Aqueous humor dynamics: a review. Open Ophthalmol J. 2010;4(1):52-9.

21. Ahmad S, et al. The dynamics of aqueous humor outflow-a major review. US Ophthalmic Rev. 2014;07(02):137.

22. Tripathi, et al. Trabecular cells express the TGF-beta 2 gene and secrete the cytokine. Exp Eye Res. 1994;58(5):523-8.

23. Yun, et al. Proteins secreted by human trabecular cells. Glucocorticoid and other effects. Investig Ophthalmol Vis Sci. 1989;30(9):2012-22.

24. David, et al. Epidemiology of intraocular pressure in a population screened for glaucoma. Br J Ophthalmol. 1987;71(10):766-71.

25. Klein BEK, et al. Intraocular pressure in an American community. The Beaver Dam Eye Study. Investig Ophthalmol Vis Sci. 1992;33(7):2224-8.

26. Last JA, et al. Elastic modulus determination of normal and glaucomatous human trabecular meshwork. Investig Opthalmol Vis Sci. 2011;52(5):2147.

27. Ashpole NE, et al. Shear stress-triggered nitric oxide release from Schlemm's canal cells. Investig Ophthalmol Vis Sci. 2014;55(12):8067-76.

28. Chang J, et al. Role of nitric oxide in murine conventional outflow physiology. Am J Physiol Cell Physiol. 2015;309(4):C205-14.

29. Michel T, Loscalzo J. Nitroglycerin and nitric oxide-a rondo of themes in cardiovascular therapeutics. N Engl J Med. 2015;373(18):1788-9.

30. Arnold W, et al. Nitric oxide activates guanylate cyclase and increases guanosine $3^{\prime}: 5^{\prime}$-cyclic monophosphate levels in various tissue preparations. Proc Natl Acad Sci. 1977;1977:3203-7.

31. Koshland E Jr. The molecule of the year. Science. 1992;258(5090):1861.

32. Furchgott RF, Zawadzki JV. The obligatory role of endothelial cells in the relaxation of arterial smooth muscle by acetylcholine. Nature. 1980;288(5789):373-6.

33. Cherry, et al. Role of endothelial cells in relaxation of isolated arteries by bradykinin. Proc Natl Acad Sci. 1982;79(6):2106-10.

34. Furchgott, et al. Endothelial cells as mediators of vasodilation of arteries. J Cardiovasc Pharmacol. 1984;6(1984):S336.

35. Hood JD, et al. VEGF upregulates ecNOS message, protein, and NO production in human endothelial cells. Am J Physiol. 1998;274(3 Pt 2):H1054-8.

36. Kroll J, Waltenberger J. VEGF-A induces expression of eNOS and iNOS in endothelial cells via VEGF receptor-2 (KDR). Biochem Biophys Res Commun. $1998 ; 252(3): 743-6$.

37. Garcia-Calvo M, et al. Purification and reconstitution of the high-conductance, calcium activated potassium channel from tracheal smooth muscle. J Biol Chem. 1994;269(1994):676-82.

38. Kubes P, Granger DN. Nitric oxide modulates microvascular permeability. Am J Physiol. 1992;262(2 Pt 2):H611-5.

39. Fukumura D, et al. Predominant role of endothelial nitric oxide synthase in vascular endothelial growth factor-induced angiogenesis and vascular permeability. Proc Natl Acad Sci. 2001;98(5):2604-9.

40. Antosova M, et al. Nitric oxide-important messenger in human body. Open J Mol Integr Physiol. 2012;02(03):98.

41. Anggard E. Nitric oxide: mediator, murderer, and medicine. Lancet. 1994;343(8907):1199-206.

42. Doganay S, et al. Decreased nitric oxide production in primary open-angle glaucoma. Eur J Ophthalmol. 2002;12(1):44-8.

43. Nathanson JA, McKee M. Identification of an extensive system of nitric oxide-producing cells in the ciliary muscle and outflow pathway of the human eye. Investig Ophthal Vis Sci. 1995;36(9):1765-73.

44. Schneemann A, et al. Nitric oxide/guanylate cyclase pathways and flow in anterior segment perfusion. Graefe's Arch Clin Exp Ophthalmol. 2002;240(11):936-41.

45. Dismuke $\mathrm{M}$, et al. Concentration-related effects of nitric oxide and endothelin-1 on human trabecular meshwork cell contractility. Exp Eye Res. 2014;120(2014):28-35.

46. Dismuke WM, et al. NO-induced regulation of human trabecular meshwork cell volume and 
aqueous humor outflow facility involve the BKCa ion channel. Am J Physiol Cell Physiol. 2008;294(6):C1378-86.

47. Ellis D, et al. Characterization of soluble guanylate cyclase in NO-induced increases in aqueous humor outflow facility and in the trabecular meshwork. Investig Ophthalmol Vis Sci. 2009;50(4):1808-13.

48. Nathanson JA, McKee M. Alterations of ocular nitric oxide synthase in human glaucoma. Investig Ophthalmol Vis Sci. 1995;36(9):1774-84.

49. Kang JH, et al. Endothelial nitric oxide synthase gene variants and primary open-angle glaucoma: interactions with sex and postmenopausal hormone use. Investig Opthalmol Vis Sci. 2010;51(2):971.

50. Walford G, Loscalzo J. Nitric oxide in vascular biology. J Thromb Haemost (JTH). 2003;1(10):2112-8.

51. Cheng C, et al. Shear stress affects the intracellular distribution of eNOS: direct demonstration by a novel in vivo technique. Blood. 2005;106(12):3691-8.

52. Ziegler T, et al. Nitric oxide synthase expression in endothelial cells exposed to mechanical forces. Hypertension. 1998;32(2):351-5.

53. Ethier CR, et al. Biomechanics of Schlemm's canal endothelial cells: influence on F-actin architecture. Biophys J. 2004;87(4):2828-37.

54. Hamanaka T, et al. Aspects of the development of Schlemm's canal. Exp Eye Res. 1992;55(3):479-88.

55. Ramos RF, et al. Schlemm's canal endothelia, lymphatic, or blood vasculature? J Glaucoma. 2007;16(4):391-405.

56. Stamer D, et al. eNOS, a pressure-dependent regulator of intraocular pressure. Investig Ophthalmol Vis Sci. 2011;52(13):9438-44.

57. Moncada S, et al. Nitric oxide: physiology, pathophysiology, and pharmacology. Pharmacol Rev. 1991;43(2):109-42.

58. Predescu D, et al. Constitutive eNOS-derived nitric oxide is a determinant of endothelial junctional integrity. Am J Physiol Lung Cell Mol Physiol. 2005;289(3):L371-81.

59. Overby DR, et al. The structure of the trabecular meshwork, its connections to the ciliary muscle, and the effect of pilocarpine on outflow facility in mice. Investig Opthalmol Vis Sci. 2014;55(6):3727.

60. Yamamoto, et al. Enhanced expression of nitric oxide synthase by rat retina following pterygopalatine parasympathetic denervation. Brain Res. 1993;631(1):83-8.

61. Kitamura $\mathrm{Y}$, et al. Nitric oxide-mediated retinal arteriolar and arterial dilatation induced by substance P. Investig Ophthalmol Vis Sci. 1993;34(10):2859-65.

62. Goureau O, et al. Differential regulation of inducible nitric oxide synthase by fibroblast growth factors and transforming growth factor beta in bovine retinal pigmented epithelial cells: inverse correlation with cellular proliferation. Proc Natl Acad Sci. 1993;90(9):4276-80.

63. Bredt D, et al. Localization of nitric oxide synthase indicating a neural role for nitric oxide. Nature. 1990;347(6295):768-70.

64. Deussen A, et al. L-Arginine-derived nitric oxide: a major determinant of uveal blood flow. Exp Eye Res. 1993;57(2):129-34.

65. Schmetterer L, et al. The effect of systemic nitric oxide-synthase inhibition on ocular fundus pulsations in man. Exp Eye Res. 1997;64(3):305-12.

66. Grunwald JE, et al. Effect of isosorbide mononitrate on the human optic nerve and choroidal circulations. Br J Ophthalmol. 1999;83(2):162-7.

67. Moncada S, Higgs A. The L-arginine-nitric oxide pathway. N Engl J Med. 1993;329(1993):2002-12.

68. Resch $\mathrm{H}$, et al. Endothelial dysfunction in glaucoma. Acta Ophthalmol. 2009;87(2009):4-12.

69. Rosenthal R, Fromm M. Endothelin antagonism as an active principle for glaucoma therapy. $\mathrm{Br} \mathrm{J}$ Pharmacol. 2011;162(2011):806-16.

70. Galassi, et al. Ocular haemodynamics and nitric oxide in normal pressure glaucoma. Acta Ophthalmol Scand. 2000;78(S232):37-8.

71. Henry E, Newby D, et al. Peripheral endothelial dysfunction in normal pressure glaucoma. Invest Ophthalmol Vis Sci. 1999;40(1999):1710-4.

72. Overby $\mathrm{D}$, et al. The changing paradigm of outflow resistance generation: towards synergistic models of the JCT and inner wall endothelium. Exp Eye Res. 2009;88(4):656-70.

73. Schuman J, et al. Nitrovasodilator effects on intraocular pressure and outflow facility in monkeys. Exp Eye Res. 1994;58(1):99-105.

74. Källberg $\mathrm{M}$, et al. Endothelin-1, nitric oxide, and glutamate in the normal and glaucomatous dog eye. Vet Ophthalmol. 2007;10(s1):46-52. 
75. Galassi F, et al. Nitric oxide proxies and ocular perfusion pressure in primary open angle glaucoma. Br J Ophthalmol. 2004;88(6):757-60.

76. Henry E, et al. Peripheral endothelial dysfunction in normal pressure glaucoma. Investig Ophthalmol Vis Sci. 1999;40(8):1710-4.

77. Henry E, et al. Altered endothelin-1 vasoreactivity in patients with untreated normal-pressure glaucoma. Investig Ophthalmol Vis Sci. 2006;47(6):2528-32.

78. Gresele $\mathrm{P}$, et al. Hyperglycemia-induced platelet activation in type 2 diabetes is resistant to aspirin but not to a nitric oxide-donating agent. Diabetes Care. 2010;33(6):1262-8.

79. Colwell J, Nesto R. The platelet in diabetes. Diabetes Care. 2003;26(7):2181-8.

80. Khoobehi B, et al. Enhanced oxygen saturation in optic nerve head of non-human primate eyes following the intravitreal injection of NCX 434, an innovative nitric oxide-donating glucocorticoid. J Ocul Pharmacol Ther. 2011;27(2):115-21.

81. Araie $\mathrm{M}$, et al. Evaluation of the effect of latanoprostene bunod ophthalmic solution, $0.024 \%$ in lowering intraocular pressure over $24 \mathrm{~h}$ in healthy Japanese subjects. Adv Ther. 2015;32(11):1128-39.
82. Weinreb R, et al. A randomised, controlled comparison of latanoprostene bunod and latanoprost $0.005 \%$ in the treatment of ocular hypertension and open angle glaucoma: the VOYAGER study. Br J Ophthalmol. 2014;99(6):305908.

83. Liu J, et al. Efficacy of latanoprostene bunod ophthalmic solution $0.024 \%$ compared with timolol maleate ophthalmic solution $0.5 \%$ in lowering IOP over 24 hours in subjects with open angle glaucoma or ocular hypertension (CONSTELLATION). Investig Ophthalmol Vis Sci. 2014;55(13):3549.

84. Weinreb $\mathrm{R}$, et al. Latanoprostene bunod $0.024 \%$ versus timolol maleate $0.5 \%$ in subjects with open-angle glaucoma or ocular hypertension the APOLLO study. Ophthalmology. 2016;123(5):965-73.

85. Medeiros F, et al. Comparison of latanoprostene bunod $0.024 \%$ and timolol maleate $0.5 \%$ in open-angle glaucoma or ocular hypertension: the LUNAR study. Am J Ophthalmol. 2016;168(2016):250-9.

86. The Group F, et al. Long-term safety and efficacy of latanoprostene bunod $0.024 \%$ in Japanese subjects with open-angle glaucoma or ocular hypertension: the JUPITER study. Adv Ther. 2016;33(9):1612-27. 